\title{
ANTIOXIDANT STATUS, OXIDATIVE STRESS AND LIPID PROFILE IN ESSENTIAL HYPERTENSIVE MEN
}

Shantha Kumari. N, Hemalatha, Sheethal. K. C, Shwetha, Rashmi. T.M.
1. Assistant Professor. Department of Biochemistry, Dr. B. R. Ambedkar Medical College, Kadugondanahalli, Bangalore.
2. HOD \& Professor. Department of Biochemistry, Dr. B. R. Ambedkar Medical College, Kadugondanahalli, Bangalore.
3. Tutor. Department of Biochemistry, Dr. B. R. Ambedkar Medical College, Kadugondanahalli, Bangalore.
4. Tutor. Department of Biochemistry, Dr. B. R. Ambedkar Medical College, Kadugondanahalli, Bangalore.
5. Lecturer. Department of Biochemistry, Dr. B. R. Ambedkar Medical College, Kadugondanahalli, Bangalore.

\section{CORRESPONDING AUTHOR:}

Dr. Shantha Kumari. N,

Assistant Professor.

Department of Biochemistry,

Dr. B. R. Ambedkar Medical College,

Kadugondanahalli, Bangalore-560045.

E-mail: drshanthakumari17@gmail.com

ABSTRACT: Hypertension is a major health burden and coexists of with imbalance in antioxidants and lipid profile. This study is done to evaluate the total antioxidant status and oxidative stress like Malondialdehyde (MDA), catalase, superoxide dismutase (SOD), uric acid and correlate with lipid profile among hypertensive men. Significant increase in MDA, uric acid and dyslipidemia was found among hypertensive men. Also total antioxidant status and other antioxidants like SOD, catalase was found to be decreased in hypertensive men. Hence it is concluded that lipid peroxidation occur in hypertension which leads to endothelial dysfunction, renal blood flow alteration and tissue damage. As a result of which dyslipidemia, hyperuricemia and decrease in anti oxidants are seen. Hence decrease in antioxidants is a useful marker for antioxidant therapy to prevent organ damage among hypertensive.

INDRODUCTION: Hypertension is a major health burden and is associated with atherosclerosis directing towards pathophysiology of many cardiovascular disease like stroke, ischemic heart disease, congestive cardiac failure till cardiovascular death (1).

Etiology of hypertension is multifactorial complex and poorly understood. There is a association of salt intake with blood pressure, which suggest us to overlook the role of magnesium, calcium, potassium in BP regulation (2).Calcium and lipid accumulate in media and bind to arterial wall elastin, resulting in thin lamina, fragmented and poorly demarcated media. Undifferentiated cells migrate through lamina to intima, proliferate connective tissue with thickening and fibrosis of intima, leading to arterial wall thickness and stiffness. In addition to structural changes, there is increased level of noradrenalin and sympathetic activity due to baroreceptor dysfunction (3).

Hypertension is characterized by increased resistance to blood flow and damage endothelium. Damaged endothelial cells are site of platelet aggregation and fibrinogen deposition leading to thrombus formation. This together with hypoxia weakens the vessel wall and leads to hemorrhage, necrosis and organ dysfunction (4).

There is a loss of balance between oxidative stress and antioxidant status is seen in hypertension. This leads to tissue damage through lipid peroxidation anion free radicals. These 
reactive oxygen species are controlled by wide spectra of non enzymatic and enzymatic antioxidants (5).

Reactive oxygen species (ROS) is a integral part of human metabolism, now been incriminated in aging and more than 100 disease states. Antioxidant systems counteract this damage which includes enzymes like superoxide dismutase (SOD), catalase (CAT), glutathione peroxidase; macromolecules like ceruloplasmin, ferritin, albumin; small molecules like ascorbic acid, uric acid, $\beta$ carotene, $\alpha$ tocopherol, reduced glutathione etc (6). These are present in all organisms, which increase on cellular death of affected tissues. ROS is increased which affects antioxidants reaction catalyzed by ROS scavenging enzymes. A defect in ROS scavenging system has been reported in atherosclerosis (7). ROS mediate various signaling pathways that underline vascular inflammation in atherogenesis; from initiation of fatty streak through lesion progress till plague rupture (8). Consequence of over production of critical nonlipid macromolecules affects genomic and mitochondrial DNA (9).

The present study is aimed to assess the association of antioxidant status, oxidative stress hyperuricemia and dyslipidemia among elderly men with essential hypertension. We assessed the antioxidant as a marker, in context of traditional dyslipidemia and hyperuricemia.

METHODS AND MATERIALS : Ninety six male subjects between 35 - 65 yrs were included in the study out of which 46 healthy subjects were considered as normotensive (control group) with $\mathrm{BP} \leq 120 / 80 \mathrm{mmHg}$. Cases were age matched 51 subjects enrolled from OPD, dept of medicine Dr. B. R. Ambedkar Medical College. Cases included were hypertensives with average blood pressure $\geq 140 / 90 \mathrm{mmHg}$ for a period more than $10 \mathrm{yrs}$, as defined by JNC $7^{\text {th }}$ criteria (10). Patients with renal disorders, diabetes mellitus, liver disorder, gout, familial hyperlipidemia were excluded. Also patients those who were on anti-oxidants, vasoactive medicine, lipid lowering statins were excluded. Informed consent was taken from each patient and study was approved by institutional ethical committee.

A volume of $5 \mathrm{ml}$ of fasting venous blood sample was collected with aseptic precautions. Blood samples were allowed to clot. Serum was then separated and analyzed for lipid profile, uric acid, Malondialdehyde (MDA), SOD, CAT, and Total antioxidant status (TAS). Total cholesterol, high density lipoprotein cholesterol (HDL-C), Triglyceride (TG), uric acid was estimated using Erba assay kits. Low density lipoprotein cholesterol (LDL-C) was calculated by Freidewalds formula (11). Very low density lipoprotein cholesterol (VLDL- C) was calculated by formula TG/5. MDA was measured using thio-barbituric acid (TBA). The mixture was heated at $100 \mathrm{C}$ for $15 \mathrm{~min}$, allowed to cool and centrifuged at 5000rpm. The OD of the supernatant was observed at $535 \mathrm{~nm}$ (12). Catalase was measured using $\mathrm{H}_{2} \mathrm{O}_{2}$-phosphate buffer and OD measured at $240 \mathrm{~nm}$ (13). SOD was measured using Nitro blue tetrazolium dye, which gets reduced by super-oxide anions, generated by peroxidation of hydroxylamine hydrochloride and OD measured at $560 \mathrm{~nm}$ (14). Total anti-oxidant status was assayed by measuring FRAP (ferric reducing activity of plasma). FRAP uses antioxidant as reductant in a redox linked colorimetric method. At lower $\mathrm{pH}$, reduction of a ferric tripyridyltriazine complex to a ferrous form produces an intense blue colour, which is measured at 593nm (15).

Statistical software was used to analyze data. Microsoft word and excel have been used to generate table. Student $t$ test, Chi-square and Fischer exact test has been used to find the significance of various parameters among cases and controls.

RESULTS: Our study showed significant increase in Triglyceride, total cholesterol, LDL-C, VLDLC, MDA, uric acid, whereas HDL-C, total antioxidants, SOD, CAT were decreased among hypertensive compared to controls, marking ongoing oxidative stress in hypertensive. 
Table: Comparison of study variables in test and controls studied

\begin{tabular}{|l|c|c|c|}
\hline \multicolumn{1}{|c|}{ variables } & Test group & Control group & P value \\
\hline Age in years & $49.33 \pm 10.01$ & $50.71 \pm 10.06$ & 0.499 \\
\hline SBP(mmHg) & $158.15 \pm 10.46$ & $121.39 \pm 8.03$ & $<0.001^{* *}$ \\
\hline DBP(mmHg) & $102.15 \pm 6.16$ & $81.08 \pm 4.70$ & $<0.001^{* *}$ \\
\hline Triglycerides (mg/dl) & $207.13 \pm 60.94$ & $145.43 \pm 62.90$ & $<0.001^{* *}$ \\
\hline Total cholesterol (mg/dl) & $229.33 \pm 63.22$ & $178.34 \pm 46.12$ & $<0.001^{* *}$ \\
\hline HDL(mg/dl) & $39.88 \pm 12.15$ & $52.89 \pm 13.30$ & $<0.001^{* *}$ \\
\hline LDL (mg/dl) & $146.60 \pm 59.90$ & $96.93 \pm 45.17$ & $<0.001^{* *}$ \\
\hline VLDL (mg/dl) & $41.27 \pm 12.15$ & $28.52 \pm 12.31$ & $<0.001^{* *}$ \\
\hline MDA & $2.62 \pm 11.06$ & $0.99 \pm 0.22$ & $0.070+$ \\
\hline SOD & $8.38 \pm 1.53$ & $10.88 \pm 9.25$ & $0.060+$ \\
\hline CAT & $1.23 \pm 0.61$ & $1.99 \pm 0.72$ & $<0.001^{* *}$ \\
\hline Uric acid(UA) & $7.45 \pm 1.47$ & $5.29 \pm 1.47$ & $<0.001^{* *}$ \\
\hline TAS & $589.73 \pm 105.19$ & $716.67 \pm 104.85$ & $<0.001^{* *}$ \\
\hline
\end{tabular}

DISCUSSION: Hypertensives have enhanced proximal tubular reabsorbing and depressed tubular secretion of uric acid causes hyperuricemia. Kidney responds to hyperuricemia by glomerular capillary thickening and sclerosis, tubular atrophy pigment deposits, degeneration in loops of Henle and interstitial deposit of uric acid in renal medullary tissue.

Pedro et al (5) revealed increased MDA, Uric acid, TGL, TC, LDL and decreased SOD, CAT, and Total anti oxidant status. But Kornelia et al (16) have shown that though MDA increase, SOD and CAT does not decrease with age or hypertension. Lantos et al (17) have found that TAS values are below normal range in hypertensives patients and values increased gradually following antihypertensive therapy. Russo et al (18) have shown that essential hypertension was associated with greater than normal lipid peroxidation and imbalance in antioxidant status, suggesting that oxidative stress is important in pathogenesis of arterial damage. Moriel et al (19) showed that lipid and water soluble antioxidants level are lower in hypercholesterolemic and hypertensives compared to normal subjects.

Dyslipidemia has high influence on hypertension. Hypercholesterolemia influence adrenergic stimulation and outcome of target organ damage is more in dyslipidemic hypertensive. LDL-C is vasoconstrictive, mitogenic, pro-inflammatory and thrombogenic. Hypertriglyceridemic hypertensive is indication of insulin resistance accompanied by modified vascular reactivity as well as elevated catecholamine and adrenocorticotropic hormone. Lipoproteins also strongly contribute to atherogenesis, playing a relevant role in hypertension. 
Apolipoprotien E polymorphism is atherogenic as it is a ligand for LDL receptor which plays a role in removal of atherogenic remnants of triglyceride rich lipoprotein and by reversing cholesterol transport in plasma and intercellular lipid transport within tissues (20).

Oxidative stress is a phenomenon which is either due to depletion of antioxidants or due to excessive production of Reactive oxygen species. Malnutrition can lead to depletion of antioxidant (21). Oxidative stress through hydrogen peroxide increase phosphorylation of tyrosine kinase, leads to binding of neutrophil on endothelium and alteration in vessel permeability (22). Oxidative stress oxidizes LDL, whose uptake is more by macrophages than non oxidative lipoprotein. Hypercholesterolemia stimulates super oxide anion leading to oxidation of LDL. Further reduction of endothelial produced nitric oxide (NO) leads to endothelial dysfunction. $\mathrm{NO}$ also leads to vasoconstriction, platelet aggregation and adhesion of neutrophils to the endothelium (23). Increased bioavailability of NO is implicated in pathomechanism of hypertension. $\mathrm{NO}$ can be scavenged by $\mathrm{O}_{2}--$ to form $\mathrm{ONOO}^{--}$, transformed to peroxy nitrous acid, which induce oxidation of endogenous compounds (16). Endothelial dysfunction cause increased platelet aggregation and leukocyte adhesion with endothelial interaction and atherogenesis, which links to process of thrombogenesis and angiogenesis (24). Uric acid stimulates vascular smooth muscle cell proliferation with activation of mitogen activated protein kinase extracellular signal regulated kinase and stimulation of platelet derived growth factor and its receptor. Smooth muscle cell proliferation is mediated by uric acid induced cyclooxygenase- 2 and thromboxanes (25). Hyperuricemia can be due to increased urate production, decreased renal excretion or both. But hypertension and hyperuricemia is partially related to decrease renal urinary urate excretion. Uric acid is a general marker of cell death, which reflects degree of circulating Xanthine oxidase (XO) activity. XO system is also important source of oxygen free radicals. Hyperuricemia is due to superoxide radical produced by enzyme XO. The superoxide produced by enzyme in parallel with uric acid by XO enzyme is a major causal factor not only for inflammatory disease, but also for oxidative stress and gene mutation (27).

Asymptomatic hyperuricemia can also be associated with decreased renal blood flow, without affecting GFR. Increased in renal and systematic hypertensive vascular disease is parallel with raising serum uric acid levels. Increased sympathetic outflow alter renal sodium handling, increased arterial pressure, decreased blood flow and decreased uric acid excretion. So uric acid raises and increase purine oxidation of reactive oxygen species and angiotensin receptor. All these lead to hypertensive vascular injury.

MDA is the end product of non enzymatic degradation of polyunsaturated fatty acids, higher levels of MDA indicates an increased in lipid peroxidation. SOD converts reactive super oxide to less reactive $\mathrm{H}_{2} \mathrm{O}_{2}$. Decrease in SOD activity would result in increased levels of super oxide anions, which is highly toxic. Catalase helps in the removal of $\mathrm{H}_{2} \mathrm{O}_{2}$, which is depressed in hypertensive. Decrease in FRAP in hypertensive is evident of decreased antioxidant status as antioxidants reduces $\mathrm{Fe}^{3+}$ to $\mathrm{Fe}^{2+}$. Increase in $\mathrm{Fe}^{3+}$ initiate lipid peroxidation and further induce cellular damage. Total cholesterol and LDL-C elevation in presence of free radicals increase the oxidation of LDL-C leading to coronary pathology in hypertensive. Thus imbalance of counteracting mechanisms to maintain vascular homeostasis, leads to vasoconstriction and impaired vascular function. These changes are effective in response to enhanced oxidative stress possibly resulting to systemic and localized inflammatory response. There is a link between dyslipidemia in hypertensive patients and impaired antioxidant efficiency. 
Hypertensive patients with abnormal lipid profile have reduced production of antioxidant, which contribute to predisposition for cardiovascular disease.

\section{BIBILOGRAPHY:}

1. C-E Chiang and $\mathrm{C}-\mathrm{H}$ Chen. Hypertension in the Asia pacific region. J Hum Hypertens 2008; 22:441-443.

2. Richard J Johnson, Jaime Herreara-Acosta, et al. Subtle Acquired renal injury as a mechanism of salt-sensitivity hypertension. N Engl J Med, 2002; 346: No.12: 913-923.

3. J Kocemba, K Kawecka-Jaszcz, et al. Isolated systolic hypertension: pathophysiology, consequences and therapeutic benefits. J Hum Hypertens 1998; 12: 621-626.

4. Ekambaram Padmini, Muniswamy UshaRani. Lipid profile alterations and oxidative stress in patients with preeclampsia: role of black tea extract on disease management. Turk J Med Sci. 2011; 41 (5):761-768.

5. J Pedro-Botet, MI Covas et al. Decreased endogenous antioxidant enzymatic status in essential hypertension. Journal of Human Hypertension2000; 14: 343-345.

6. Guohua Cao and Ronald L Prior. Comparison of different analytical methods for assessing total antioxidant capacity of human serum. Clinical Chemistry 1998; 44:6, 1309-1315.

7. Nwanjo, H. U, Oze G. et al. Oxidant stress and non-enzymatic antioxidant status in hypertensive patients in Nigeria. African journal of Biotechnology 2007; Vol.6 (14): 1681-1684.

8. Maharajan BR, Jha JC, Vishwanath P, et al. Oxidant-antioxidant status and lipid profile in hypertensive patients. J Nepal Health Res Counc. 2008 Oct; 6(13): 63-68.

9. Josep Redon, Maria R. Oliva, Carmen Tormos, et al. Antioxidant Activities and Oxidative Stress Byproducts in Human Hypertension. Hypertension. 2003; 41: 1096-1101.

10. Aram V et al. The Seventh Report of the Joint National committee on prevention, detection, evaluation and treatment of high blood pressure. JAMA. 2003; Vol 289: No.19.

11. Friedwald WT, Levy RI, Fredricson DS. Estimation of concentration of low-density lipoprotein in plasma, without use of preparative ultracentrifuge. Clin Chem 1972; 18: 499-502.

12. Frederick Bernheim, Mary L.C. Bernheim et al. The Reaction between thiobarbituric acid and the oxidation products of certain lipids. J Biol Chem. 1948; 174: 257 - 264.

13. Roland F. Beers and Irwin W. Silzer. A spectrophotometric method for measuring the breakdown of hydrogen peroxide by catalase. J Biol Chem.1952; 195: 133-140.

14. Hara P Misra and Irwin Fridovich. The role of superoxide anion in the autoxidation of epinephrine and a simple assay for superoxide dismutase. J Biol Chem. 1972; 247: 31703175.

15. Iris F.F Benzie, J.J.Strain. The Ferric reducing ability of plasma (FRAP) as a measure of "AntioxidantPower":TheFRAPassay. Analytical Biochemistry 1996; 239: 1, 70-76.

16. Kornelia Kedziora-Korna Towska et al. The markers of oxidative stress and activity of the antioxidant system in the blood of elderly patients with essential arterial hypertension. Cellular \& Molecular Biology letters.2004 Vol 9, No. 4A: 635-641.

17. Lantos J, Roth E, et al. Monitoring of plasma total antioxidant status in different diseases. Acta Chir Hung. 1997; 36(1-4): 188-189.

18. Russo C, Olivieri O, Girelli D, Faccini G, et al. Antioxidant status and lipid peroxidation in patients with essential hypertension. J Hypertens. 1998; 16(9): 1267-71. 
19. Moriel P, Plavnik FL, Zanella MT et al. Lipid peroxidation and antioxidants in hyperlipidemia and hypertension. Biol Res. 2000; 33(2): 105-12.

20. AB Bhavani, KB Sastry, N Krishna Reddy, T Padma . Lipid profile and Apolipoprotein E polymorphism in Essential Hypertension. Indian Heart J 2005; 57: 151-157.

21. Manoj K Kashyap, Vibha Yadhav et al. Different antioxidants, total antioxidant power and free radicals in essential hypertension. Molecular and Cellular Biochemistry. 2005; 277: 89-99.

22. Bourcier T, Sukhova G et al. The nuclear factor kappa-B signaling pathway participates in disregulation of vascular smooth muscle cells in vitro and in human atherosclerosis, J Biol Chem. 1997; 272: 15817-15824.

23. Georgia Vogiatzi, et al. The role of oxidative stress in atherosclerosis. Hellenic J Cardiol. 2009; 50: 402-409.

24. HS Lim and GYH Lip. Arterial stiffness in diabetes and hypertension. Journal of Human Hypertension. 2004; 18: 467-468.

25. Joshua M. Hare, Richard J. Johnson, Uric acid predicts clinical outcomes in Heart failure. Circulation. 2003; 107: 1951-1953.

26. Stefan D. Anker, Wolfram Doehner et al. Uric acid and survival in chronic heart failure: Validation and application in metabolic, functional and hemodynamis staging. Circulation. 2003; 107; 1991-1997.

27. Lily L. Wu and James T. Wu. Serum uric acid is a marker of inflammation and a marker predicting the risk of developing CVD, stroke, renal failure and cancer. J Biomed Lab Sci. 2008; Vol 20: No 1-2. 